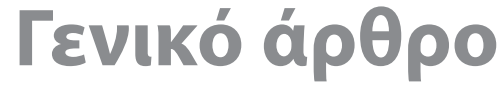 General article
}

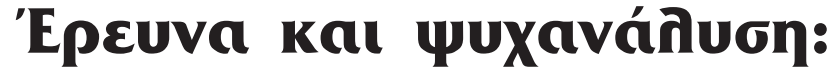

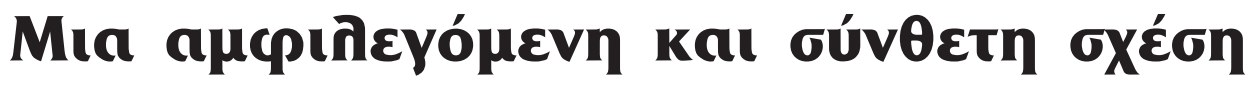

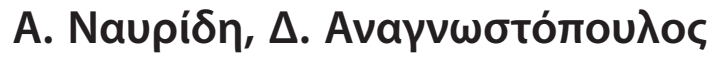

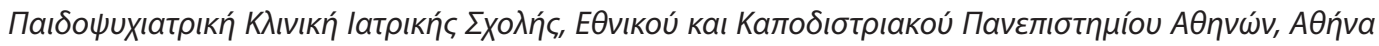 \\ Uuxıатрікń 2018, 29:359-366
}

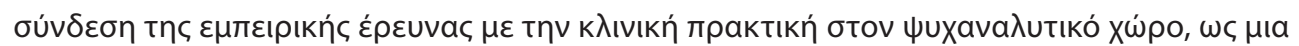

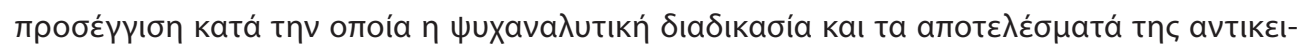

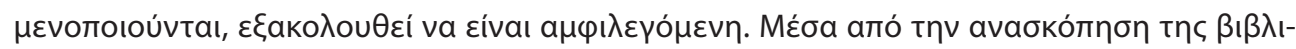

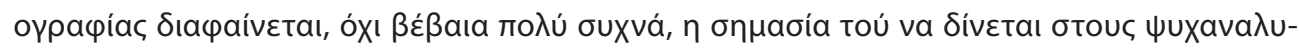

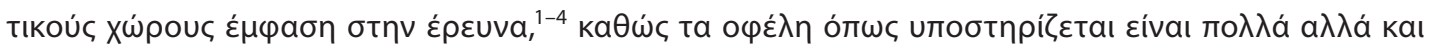

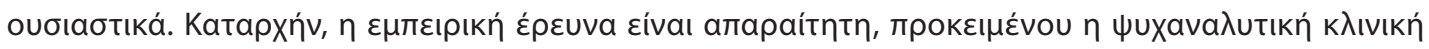

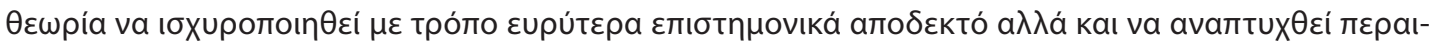

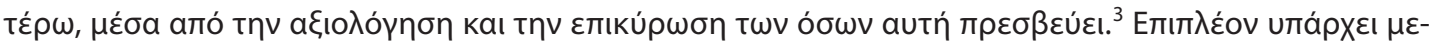

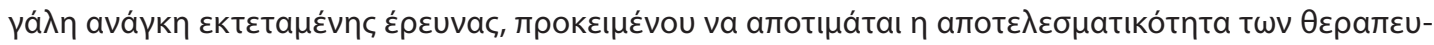

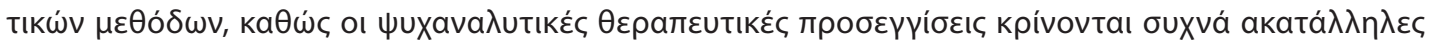

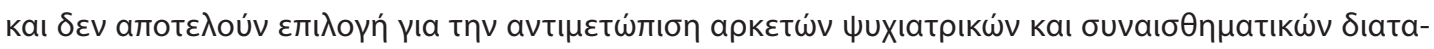

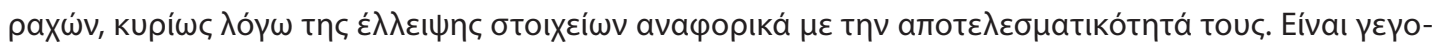

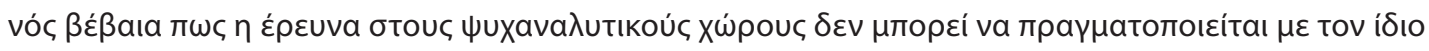

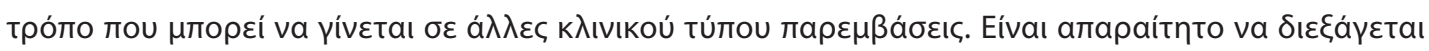

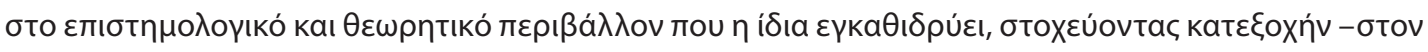

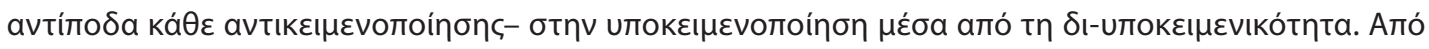

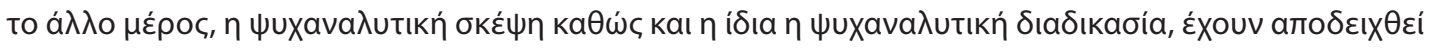

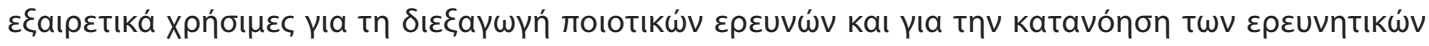

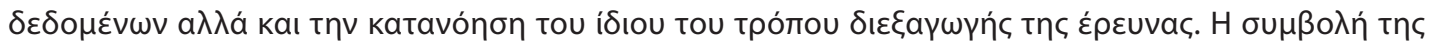

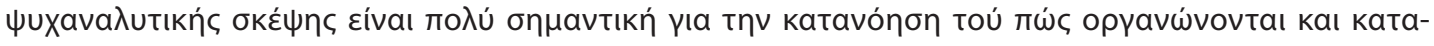

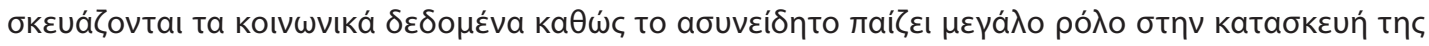

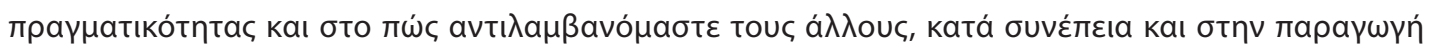




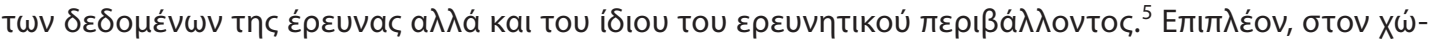

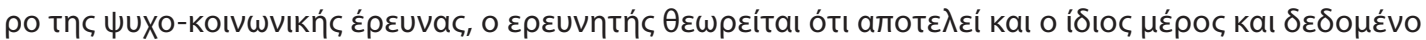

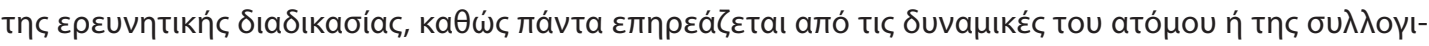

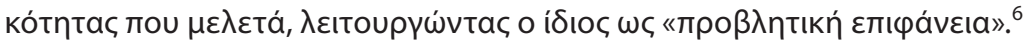

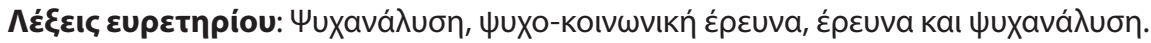

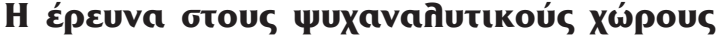

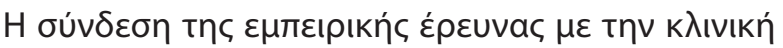

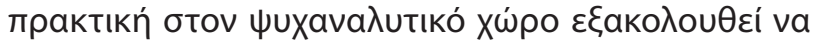

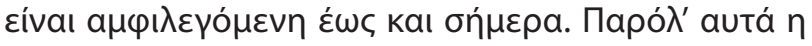

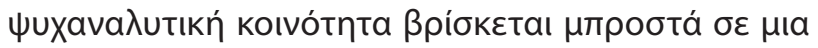

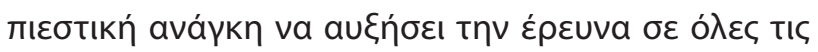

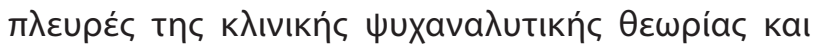

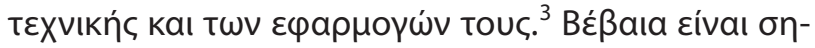

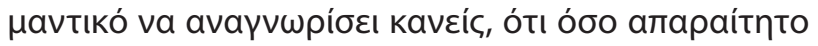

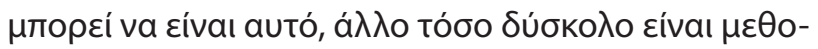

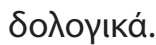

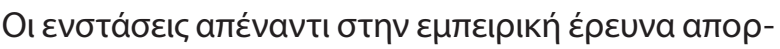

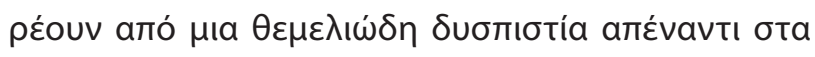

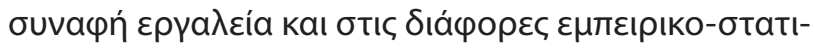

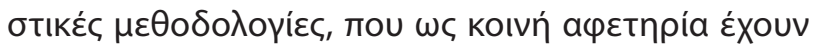

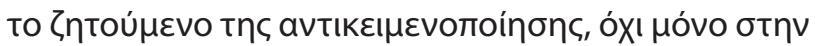

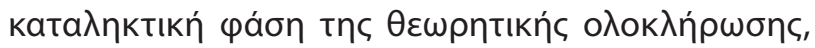

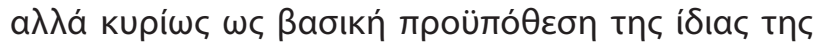

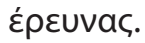

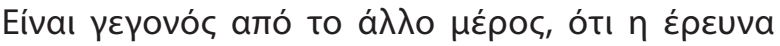

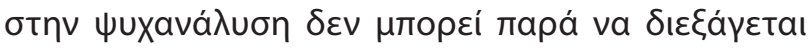

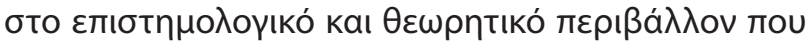

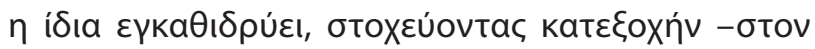

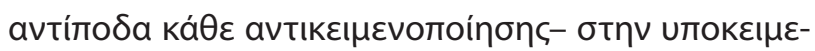

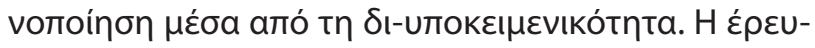

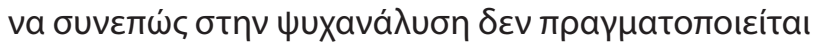

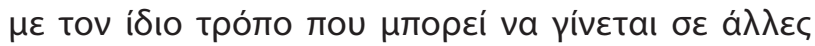

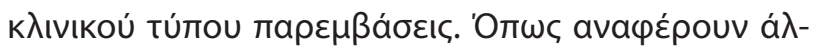

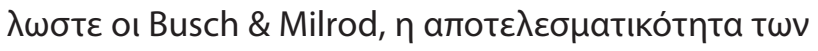

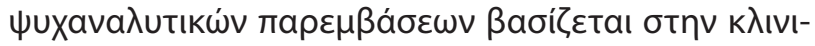

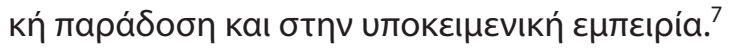

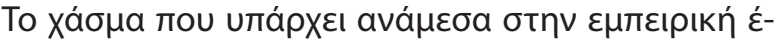

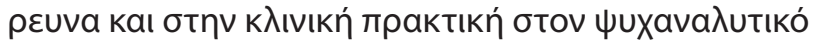

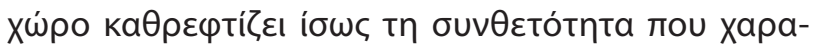

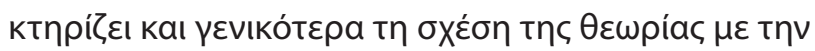

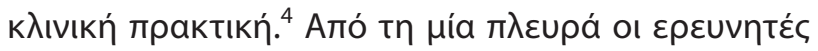

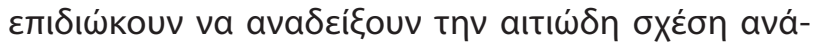

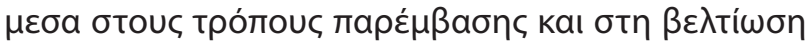

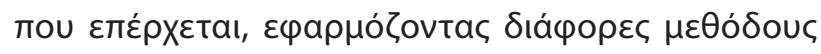
akıı入ópnons.

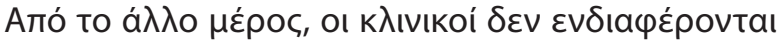

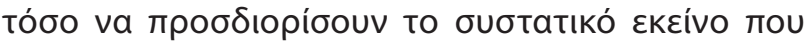

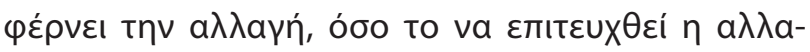

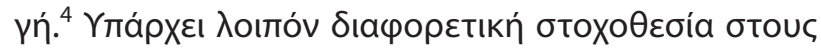

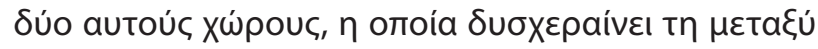

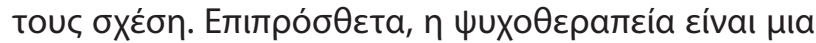

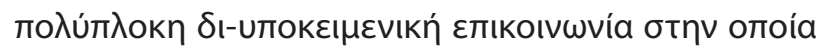

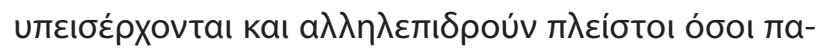

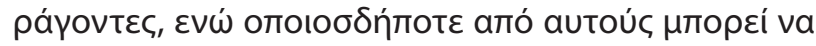

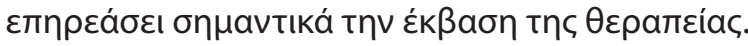

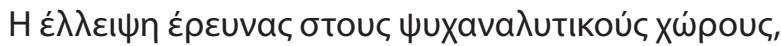
о

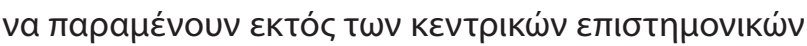

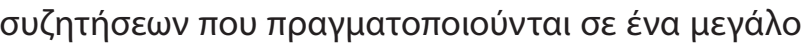

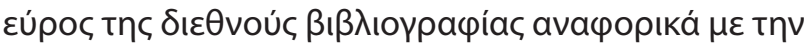

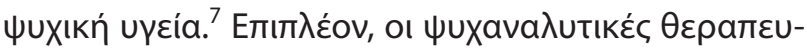

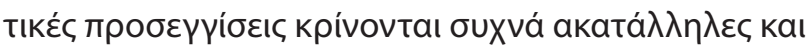

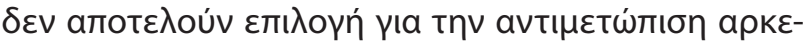

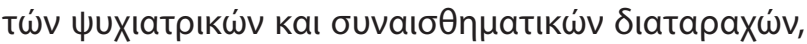

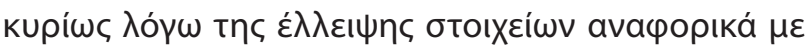

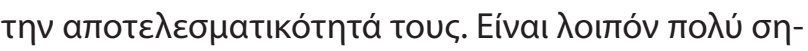

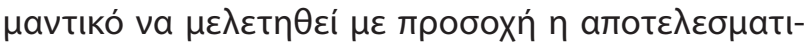

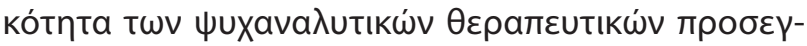

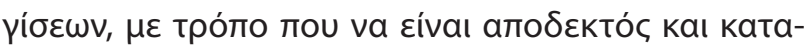

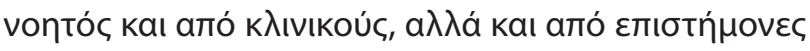

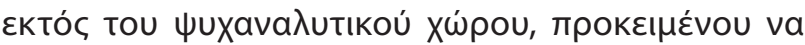

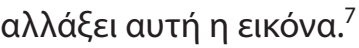

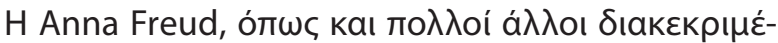

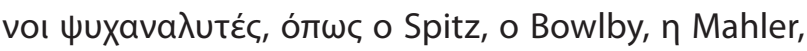

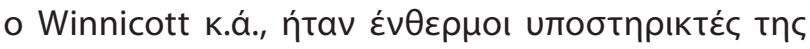

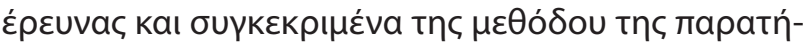

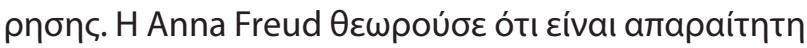

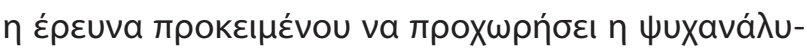

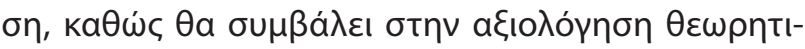

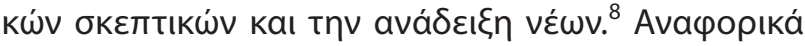

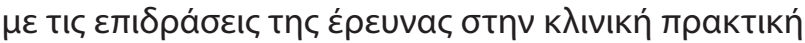




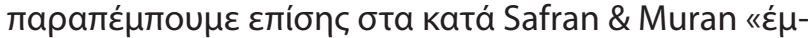

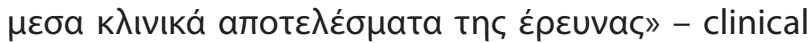

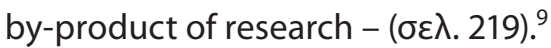

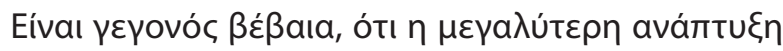

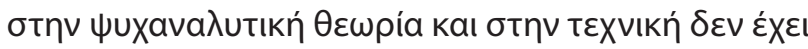

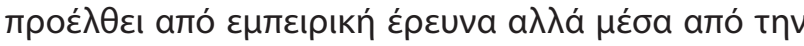

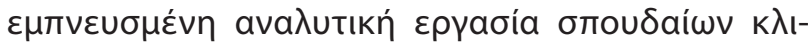

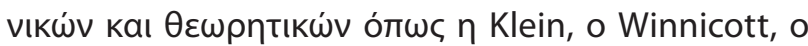

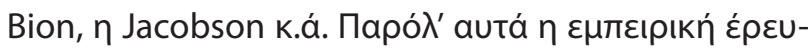

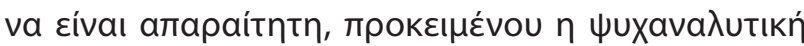

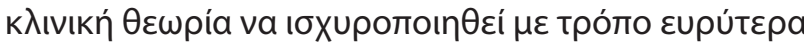

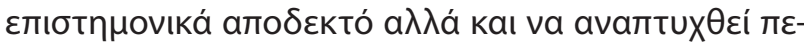

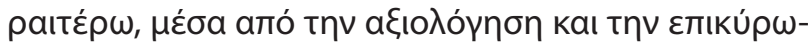

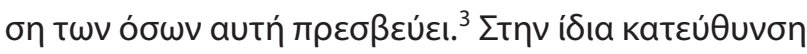

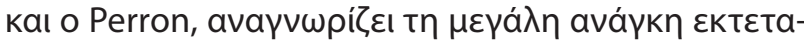

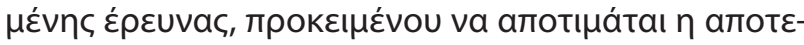

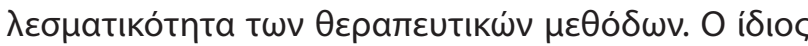

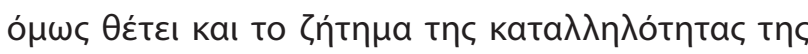

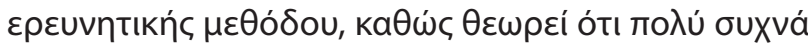

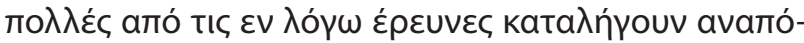

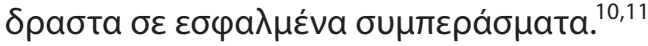

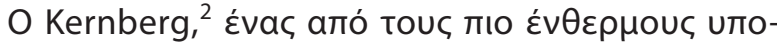

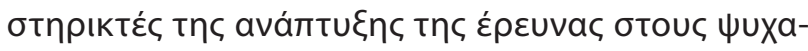

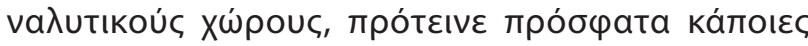

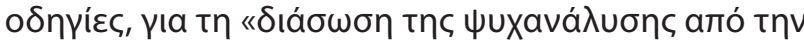

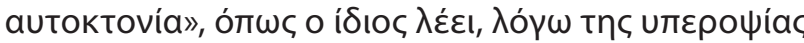

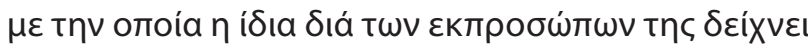

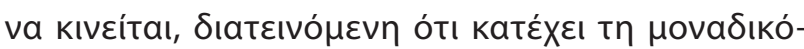

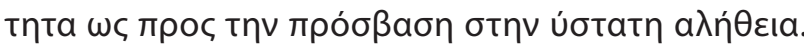

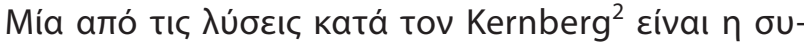

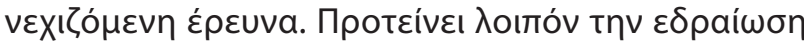

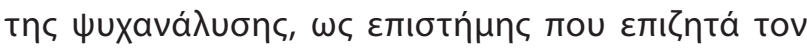

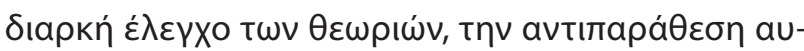

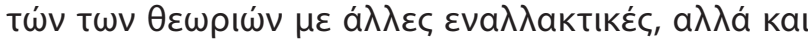

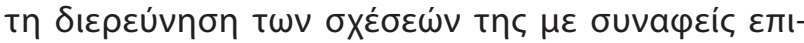

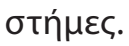

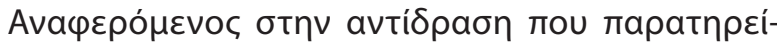

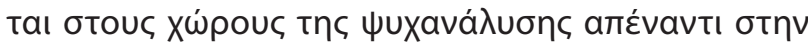

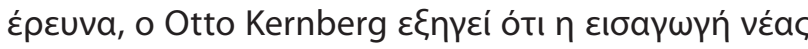

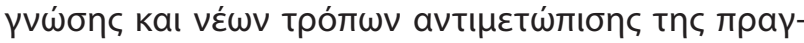

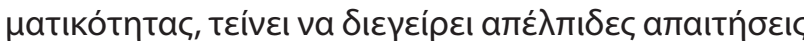

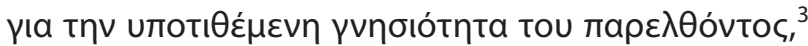

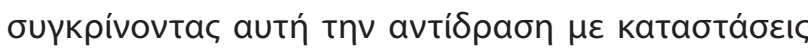

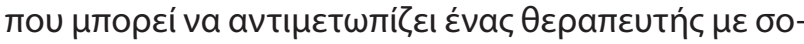

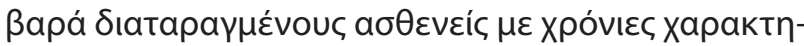

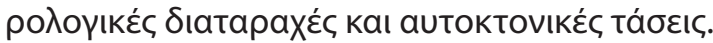

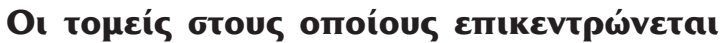

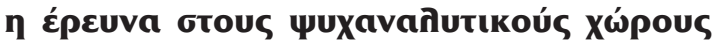

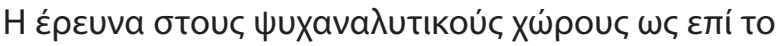

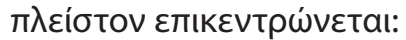

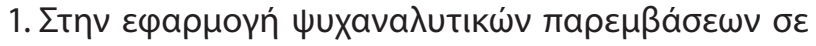

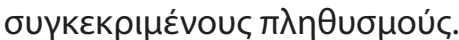

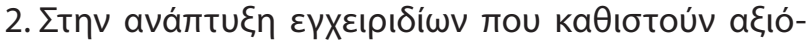

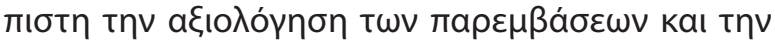

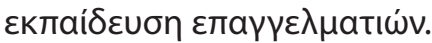

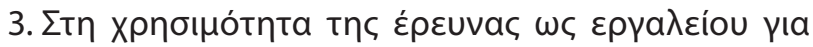

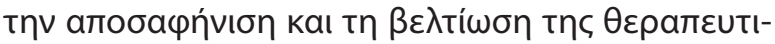

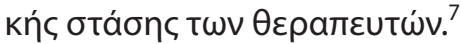

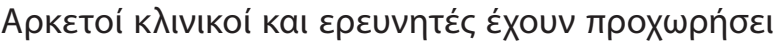

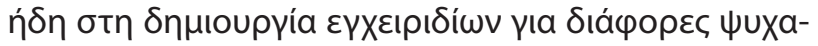

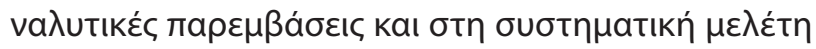

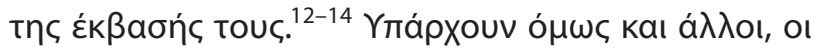
отоío

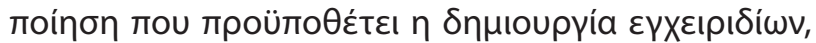

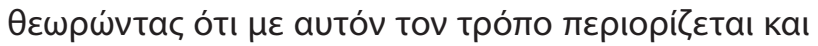

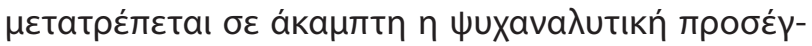

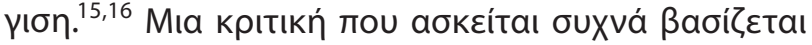

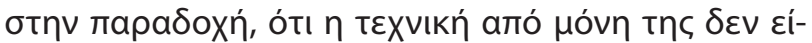

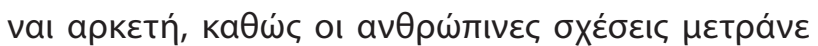

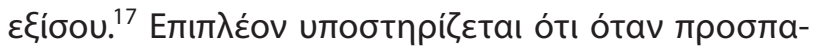

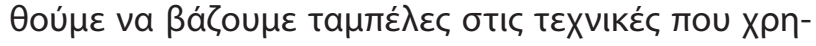

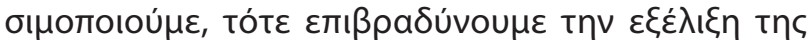

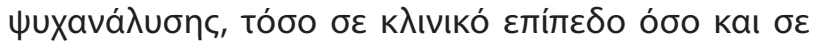
$\theta \varepsilon \omega \rho \eta$ IKó. $^{17}$

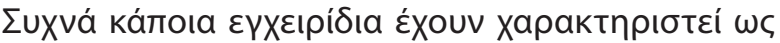

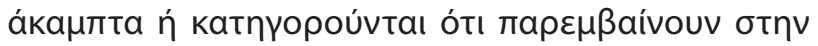

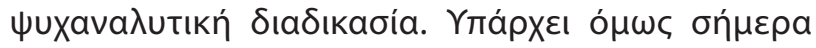

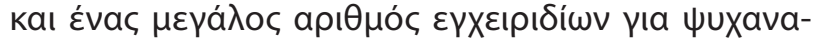

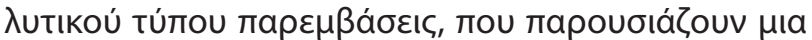

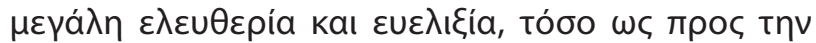

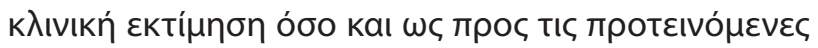

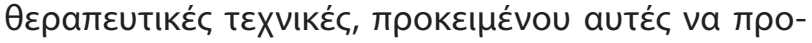

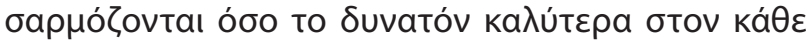

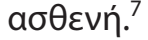

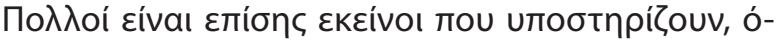

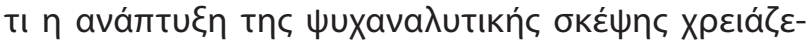

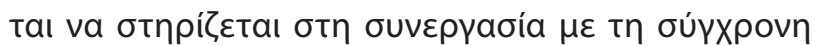

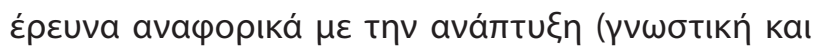

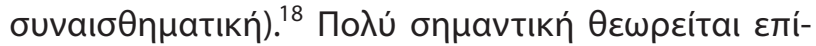

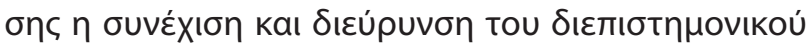

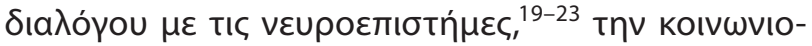




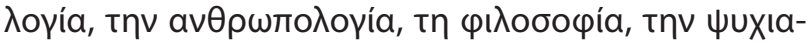

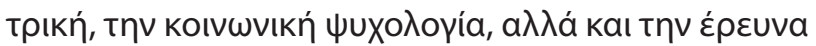

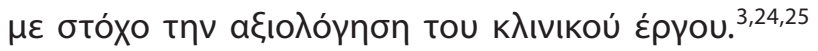

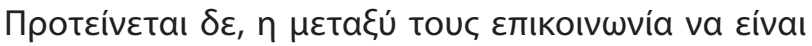

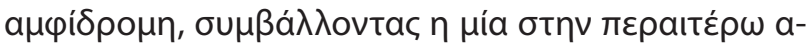

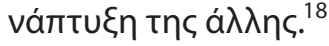

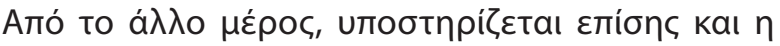

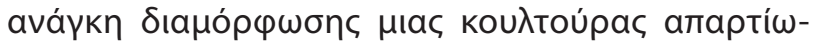

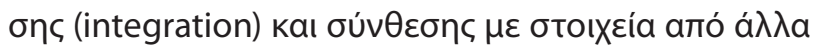

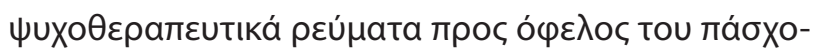

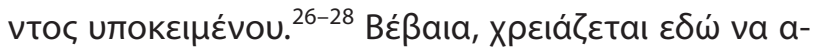

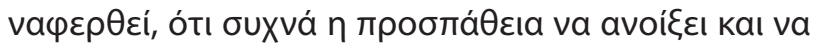

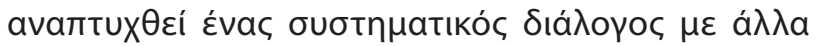

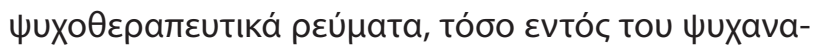

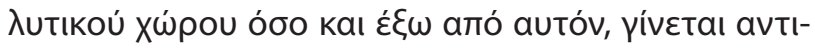

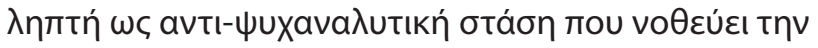

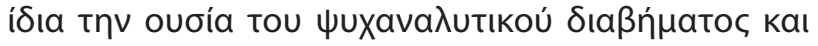

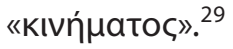

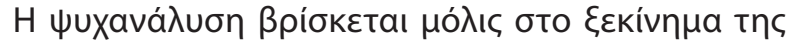

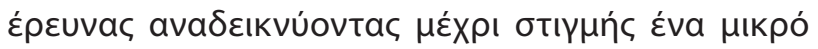

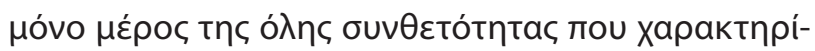

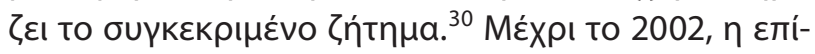

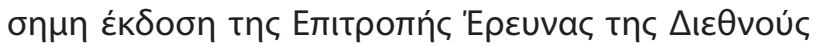

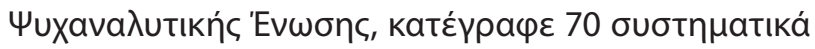

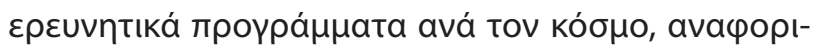

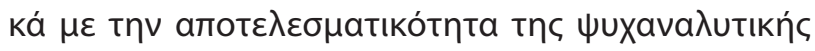

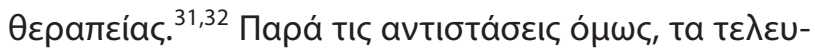

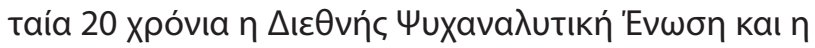

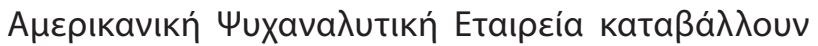

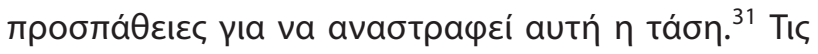

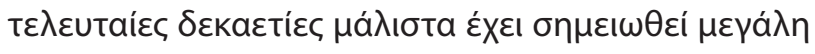

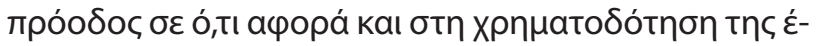

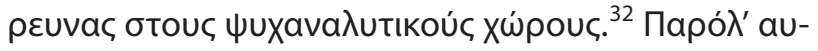

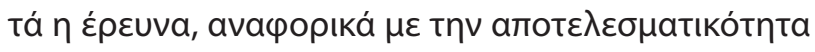

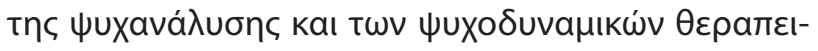

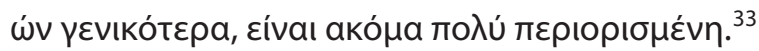

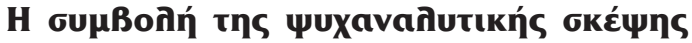 otnv épeuva}

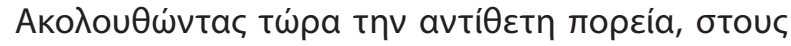

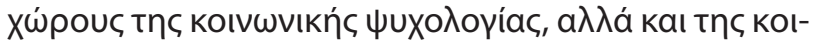

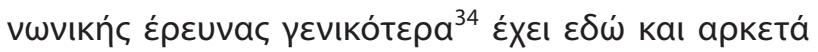

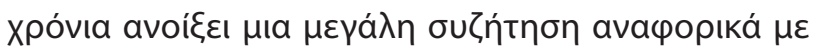

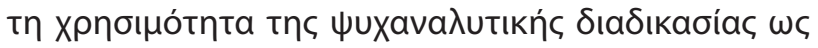

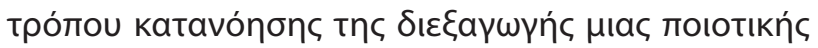

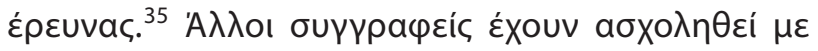

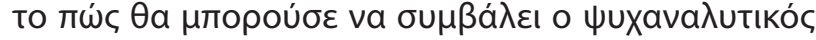

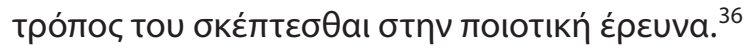

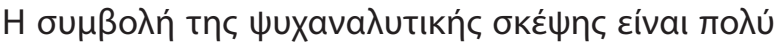

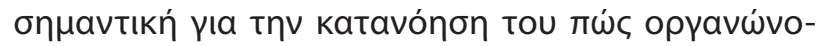

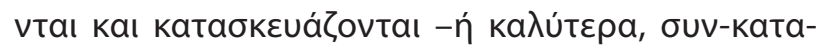

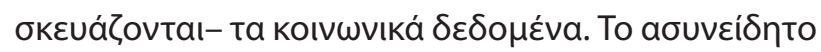

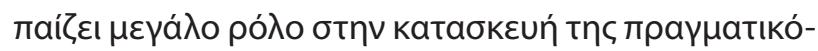

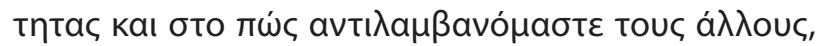

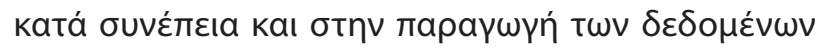

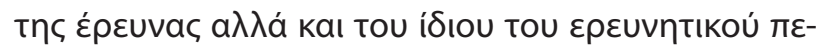

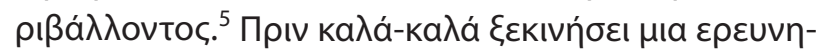

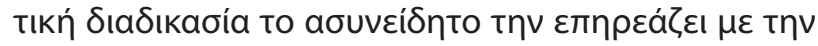

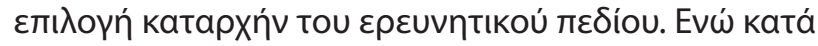

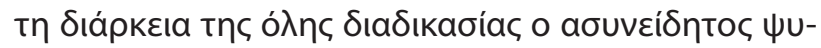

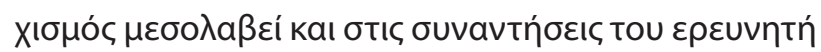

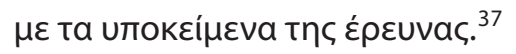

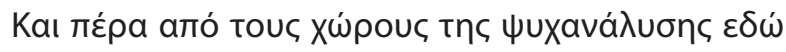

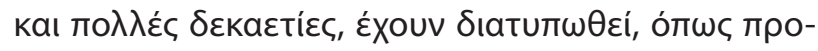

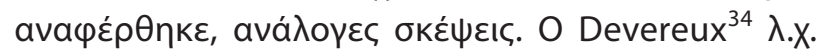

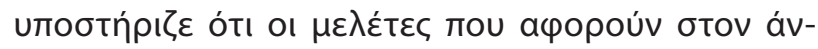

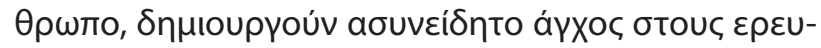

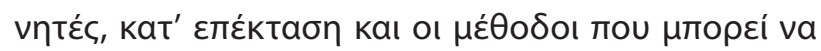

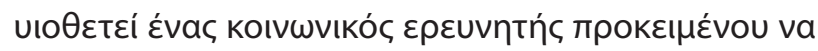

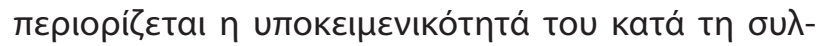

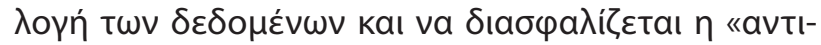

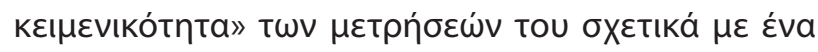

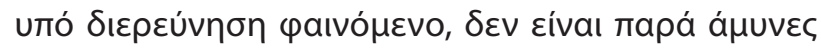

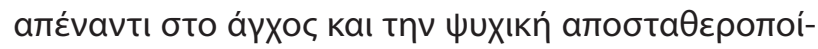

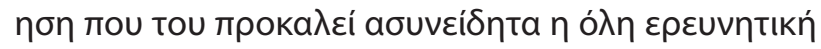

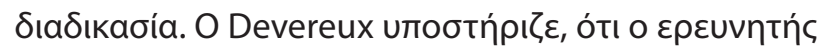

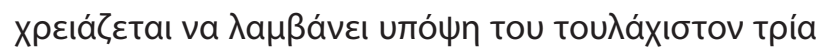
$\varepsilon i \delta \emptyset \eta \varepsilon \delta o \mu \varepsilon ́ v \omega v$ :

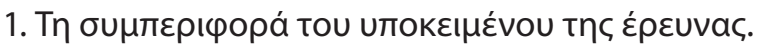

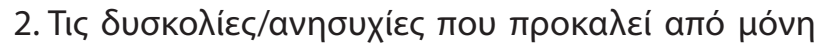

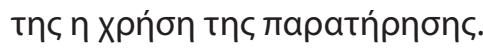

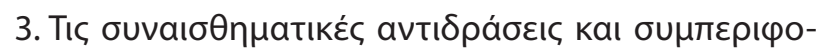

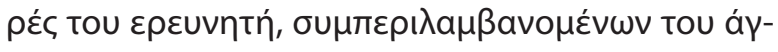

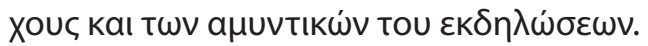

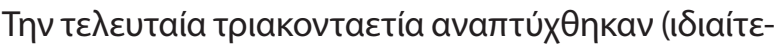

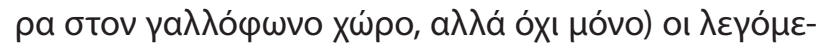

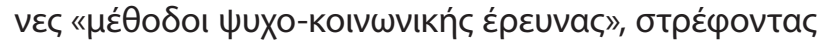

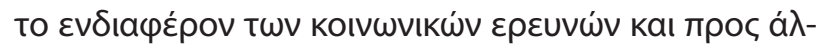

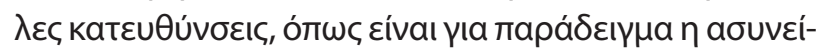

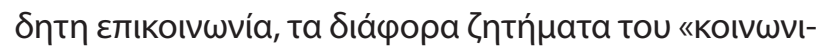

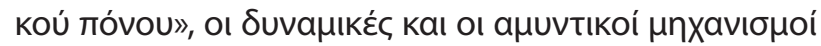

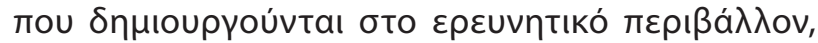




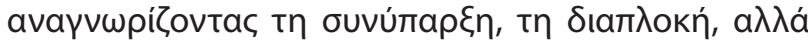

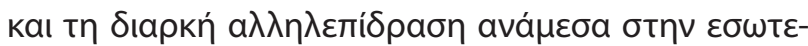

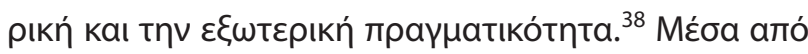

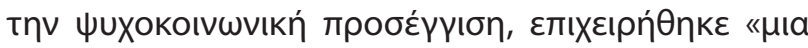

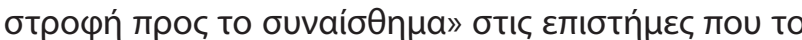

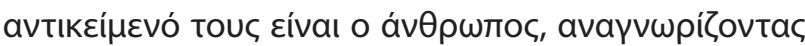

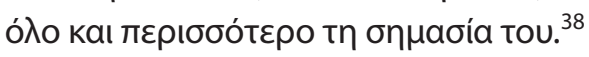

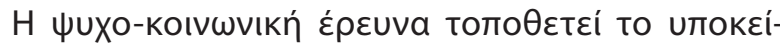

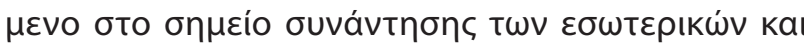

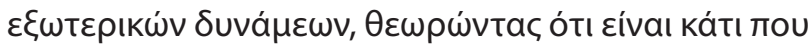

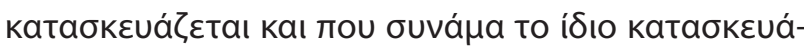

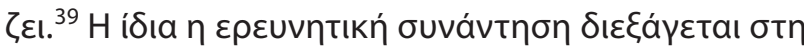

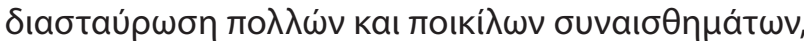

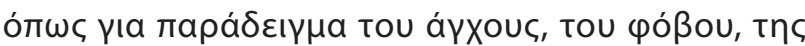

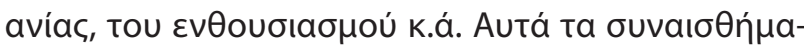

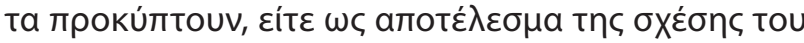

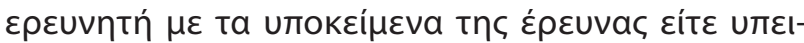

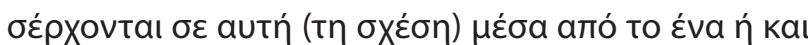

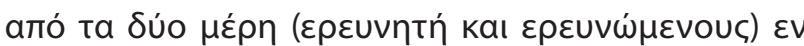
aүvoía touc. ${ }^{38}$

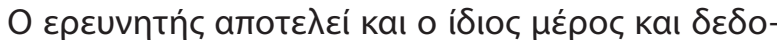

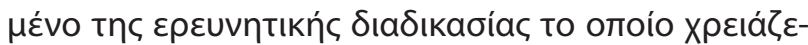

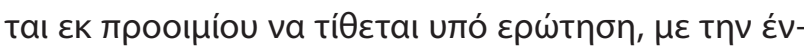

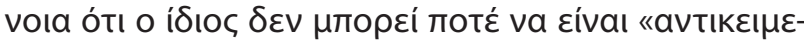

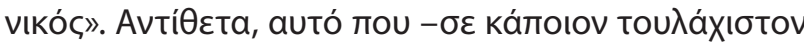

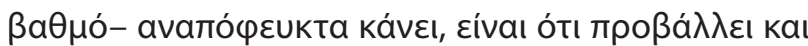

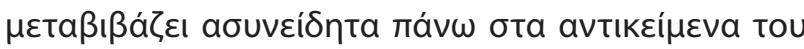

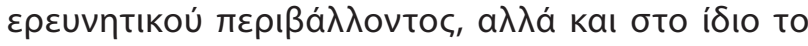

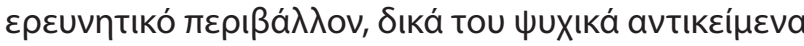

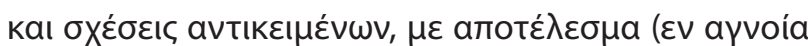

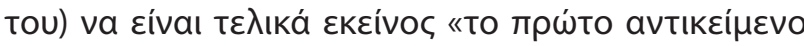

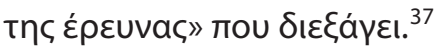

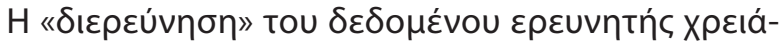

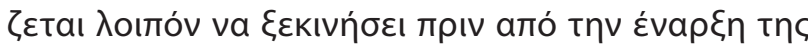

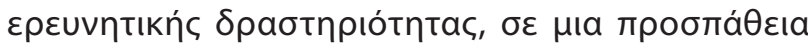

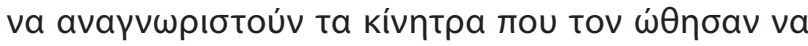

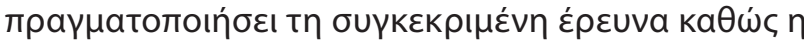

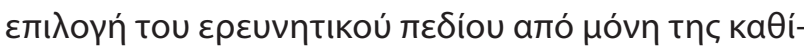

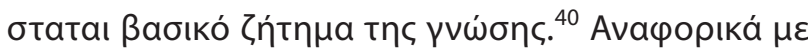

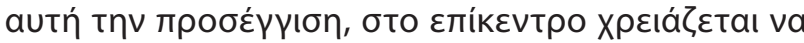

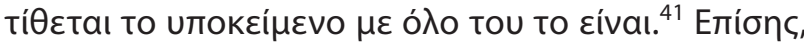

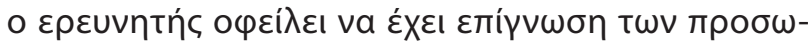

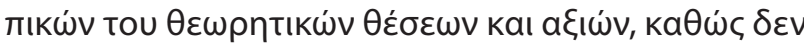

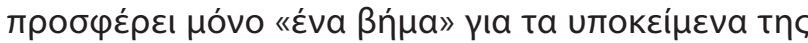

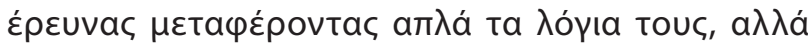

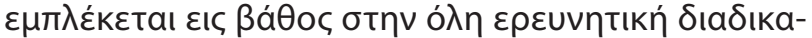
бía. ${ }^{42}$

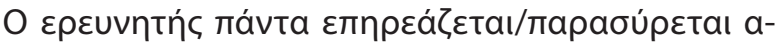

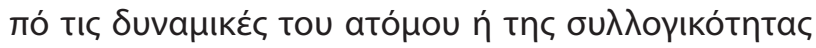

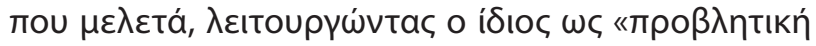

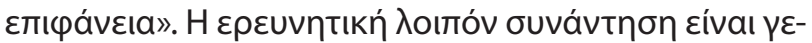

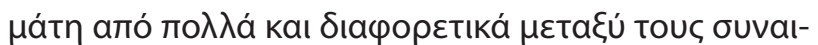

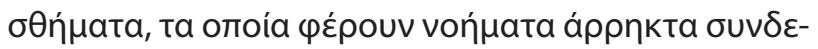

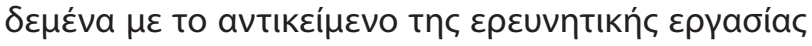

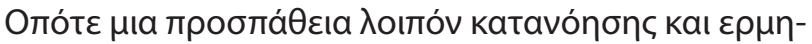

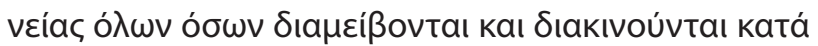

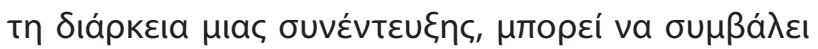

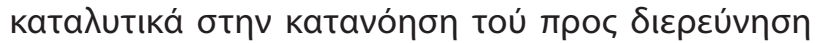

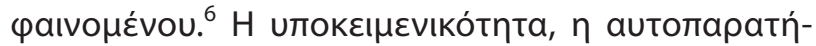

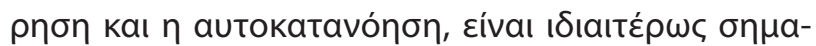

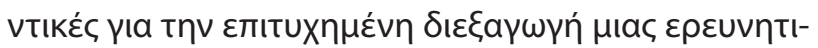

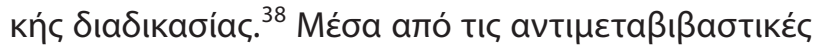

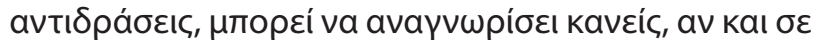

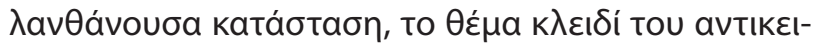

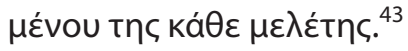

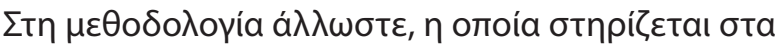

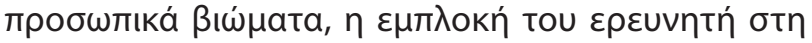

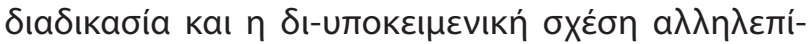

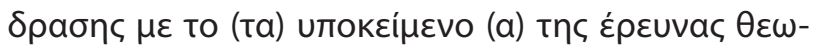

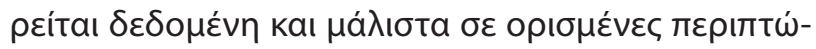

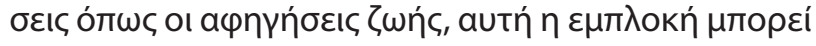

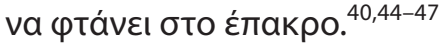

\section{Emínoyos}

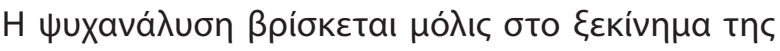

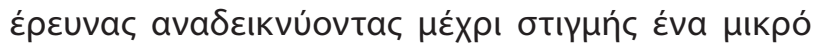

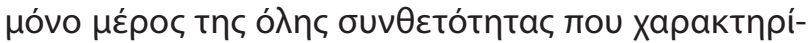

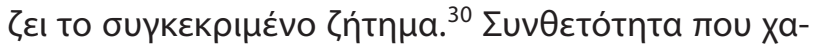

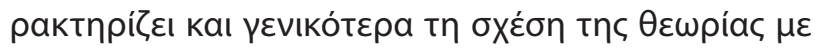

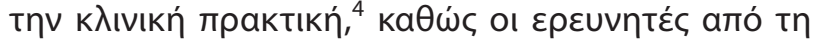

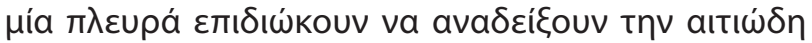

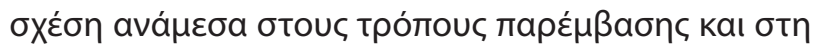

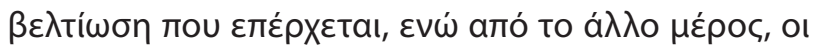

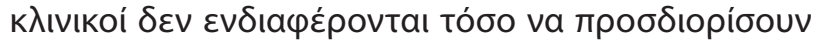

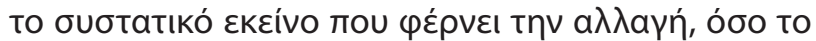

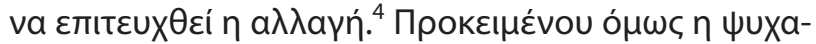

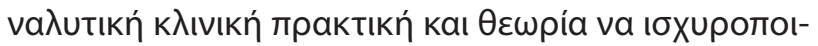

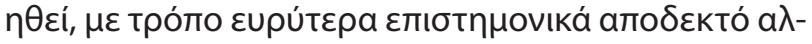

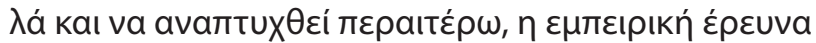

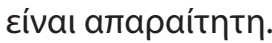




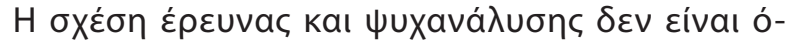

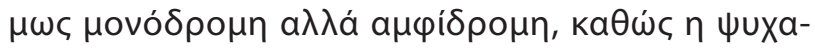

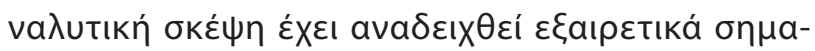

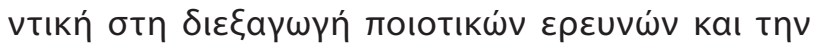

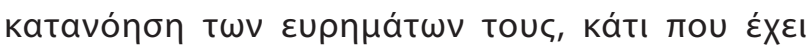

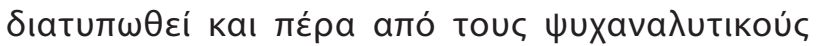

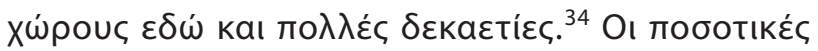

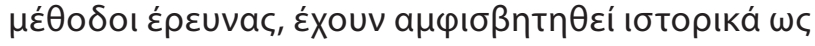

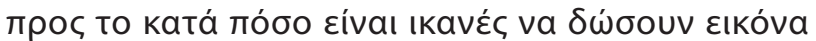

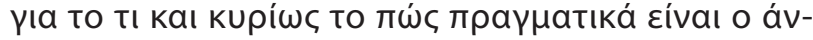

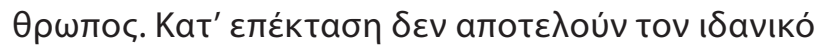

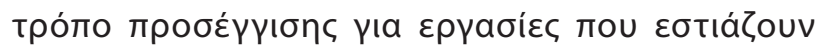

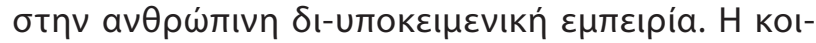

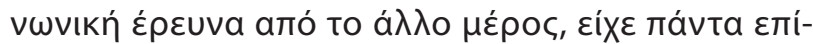

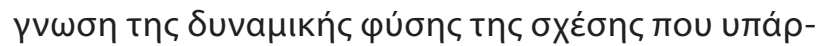

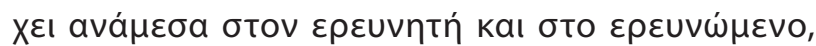

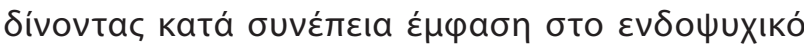

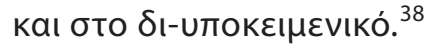

\title{
Research and psychoanalysis: A controversial and complex relationship
}

\author{
A. Navridi, D. Anagnostopoulos \\ Department of Child and Adolescent Psychiatry, National \& Kapodistrian University of Athens, Athens, Greece
}

Psychiatriki 2018, 29:359-366

The connection between empirical research and clinical practice in the psychoanalytic area, as an approach where the psychoanalytic procedure and its results get objectified, continues to be controversial. Even if it is not so frequent, the significance of giving emphasis to the psychoanalytic areas in research, is evident through the literature review, ${ }^{1-4}$ as the benefits are supported to be many and substantial. To begin with, the empirical research is necessary in order for the psychoanalytic clinical theory to be strengthened in a wider scientifically accepted way and further developed, through the evaluation and the confirmation of what it entreats. ${ }^{3}$ Moreover, there is an important need of wide research, in order to evaluate the efficacy of the therapeutic techniques, as psychoanalytic therapeutic approaches are frequently adjudged incompetent and they are not form an option of the treatment of many psychiatric and emotional disorders, mainly because of the lack of findings concerning their efficacy. It is a fact though, that research in psychoanalytic areas cannot be conducted in the same way that can be conducted in different clinical interventions. It is essential to be conducted within the epistemological and theoretical environment that the psychoanalysis itself establishes, with main purpose -avoiding every objectification- the subjectification through intersubjectivity. On the other side, both the psychoanalytic thought and the psychoanalytic procedure, have proved to be significantly useful for the conduct of qualitative research and for the understating both of research findings and the way the research itself was carried out. The contribution of the psychoanalytic thought is highly important for the understanding of how the social data are being organized and constructed, as the unconscious has a crucial role in the construction of reality and in the way we perceive others and as a result in the construction of research data and the research environment. ${ }^{5}$ In addition, in the field of psycho-social research, the researcher is perceived to be himself part and the data and of the research procedure, as he always got influenced by the dynamics of the people or the group that he studies, operating himself as a "projective surface". ${ }^{6}$

Key words: Psychoanalysis, psycho-social research, research and psychoanalysis. 


\section{Bıßntoypacpía}

1. Gunderson JG, Gabbard G. Making the case for psychoanalytic therapies in the current Psychiatric environment. J Am Psychoanal Assoc 1999, 47:679-704

2. Kernberg O. Suicide prevention for psychoanalytic institutes and societies. J Am Psychoanal Assoc 2012, 60:707-719, doi: 10.1177/0003065112449861

3. Kernberg OF. The pressing need to increase research in and on psychoanalysis. Intern J Psychoanal 2006, 87: 919-926, doi: 10.1516/46N7-ULAM-DQKR-VGRT

4. Jimenez JP. Can research influence clinical practice? Intern J Psychoanal 2007, 88:661-680, doi: 10.1516/P447-7027-L16W2362

5. Hunt J. Psychoanalytic aspects of fieldwork. Sage, London, 1989

6. Tietel E. The interview as relational space. Forum: Qualitative Social Research 2000, 1, No 3, doi: http://dx.doi.org/10. 17169/ fqs-1.2.1095

7. Busch FN, Milrod BL. The ongoing struggle for psychoanalytic research: Some steps forward. Psychoanal Psychother 2010, 24:306-314, doi: http://dx.doi.org/10.1080/02668734.2010.519 234

8. Freud A. Observations on child development. In: The Writings of Anna Freud. International UP, New York, 1951, 4:143-162

9. Safran JD, Muran JC. Towards a working alliance between research and practice. In: Talley PF, Strupp, HH, Butler SF (eds) Psychotherapy research and practice: Bridging the gap. New York, Basic Books, 1994:206-226

10. Perron R. How to do research? Reply to Otto Kernberg. Intern J Psychoanal 2006, 87:927-932

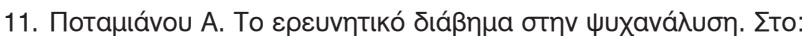

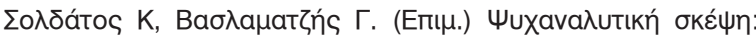

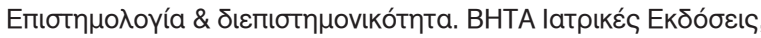
AӨńva, 2007

12. Clarkin JF, Levy KN, Lenzenweger MF, Kernberg OF. Evaluating three treatments for borderline personality disorder: A multiwave study. Am J Psychiatry 2007, 164:922-928, doi: 10.1176/ ajp.2007.164.6.922

13. Leichsenring F, Salzer S, Jaeger U, Kächele H, Kreische $R$ Leweke $F$ et al. Short-term psychodynamic psychotherapy and psychoanalytic psychotherapy and cognitive-behavioural therapy in generalized anxiety disorder: A randomized, controlled trial. Am J Psychiatry 2009, 166:875-881, doi: 10.1176/ appi.ajp.2009.09030441

14. Milrod B, Leon AC, Busch FN, Rudden M, Schwalberg M, Clarkin $\mathrm{J}$ et al. A randomized controlled clinical trial of psychoanalytic psychotherapy for panic disorder. Am J Psychiatry 2007, 164: 265-272, doi: 10.1176/ajp.2007.164.2.265

15. Blatt SJ. The effort to identify empirically supported psychological treatments and its implications for clinical research, practice and training: Commentaries on papers by Lester Luborsky and Hans H. Strupp. Psychoanal Dialog 2001, 11:635-646, doi: http://dx.doi.org/10.1080/10481881109348633

16. Busch FN, Milrod BL, Sandberg LS. A study demonstrating efficacy of a psychoanalytic psychotherapy for panic disorder:
Implications for psychoanalytic research, theory and practice. J Am Psychoanal Assoc 2009, 57:131-148, doi: https://doi. org/10.1177/0003065108329677

17. Bromberg Philip M. Something wicked this way comes: Trauma, dissociation, and conflict: The Space where psychoanalysis, cognitive science, and neuroscience overlap. Psychoanal Psychol 2003, 20:558-574, doi: http://psycnet.apa.org/doi/10. 1037/0736-9735.20.3.558

18. Bucci W. Is there language disconnected from sensory/bodily experience in speech or though? J Am Psychoanal Assoc 2012, 60:275-286, doi: https://doi.org/10.1177/0003065112441366

19. Solms M, Turnbull O. The brain and the inner world: An introduction to the neuroscience of subjective experience. Other Press, New York, 2002

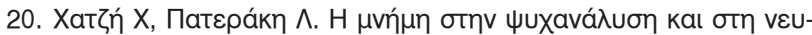

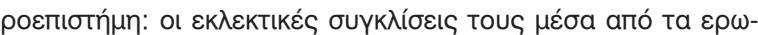

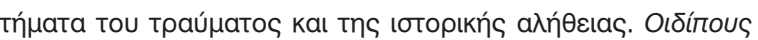
2009, 2:446-509

21. Leuzinger-Bohleber $M$, Pfeifer R. Recollecting the past in the present. Psychoanal Neurosci 2006:63-95

22. Vaslamatzis G. Framework for a new dialogue between psychoanalysis and neurosciences: is the combined neuro-psychoanalytic approach the missing link? Philosophy Ethics Human Med 2007, 2:25-31, doi: https://doi.org/10.1186/1747-5341-2-25

23. Issidorides Radovich M, Vaslamatzis G. Dialogues of psychoanalysis and neurobiology: Theoretical and therapeutic aspects. BETA Medical Publishers LtD, Athens, 2010

24. Klauber J. On the dual use of historical and scientific method in psychoanalysis. Intern J Psychoanal 1968, 49:80-88

25. Leuzinger-Bohleber M, Stuhrast U, Rüger B, Beutel M. How to study the "quality of psychoanalytic treatments" and their longterm effects on patients' well-being. Intern J Psychoanal 2003, 84:263-290, doi: 10.1516/C387-0AFM-4P34-M4BT

26. Greenberg LS, Rice LN, Elliot R. Facilitating emotional change: The moment-by-moment process. Guilford Press, New York, 1988

27. Beebe B, Lachmann FM. Representation and internalization in infancy: Three principles of salience. Psychoanal Psychol 1994, 11:127-166

28. Lancer G. Towards a common ground in psychoanalysis and family therapy: on knowing not to know. J Fam Therapy 2000, 22:61-82, doi: 10.1111/1467-6427.00138

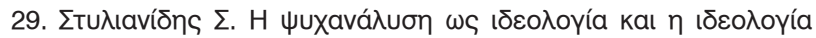

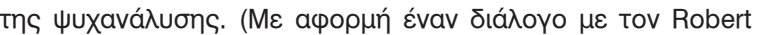
Castel)», Oıঠínouc 2010, 3:226-252

30. Scalzone F. Notes for a dialogue between psychoanalysis and neuroscience. Intern J Psychoanal 2005, 86:1405-1423, doi: 10.1516/9KGQ-67A1-RA5H-96L6

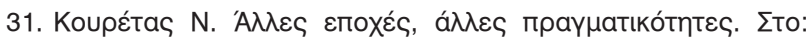

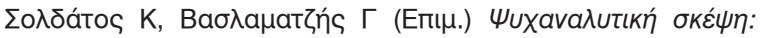

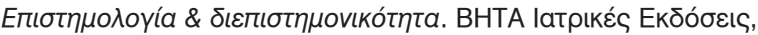
AӨńva, 2007

32. Fonagy P. An open-door review of outcome studies in psychoanalysis. UK, International Association, 2002 
33. Gerber AJ, Kocsis JH, Milrod BL, Roose SP, Barber JP, Thase $\mathrm{ME}$ et al. A quality-based review of randomized controlled trials of psychodynamic psychotherapy. Am J Psychiatry 2011, 168: 19-28, doi: https://doi.org/10.1176/appi.ajp.2010.08060843

34. Devereux G. From anxiety to method in the behavioural sciences. Moultons \& Co, 1967

35. Frosch S, Phoenix A, Pattman R, Emerson P. Interpretation and over-interpretation: Disputing the meaning of texts. Qualitative Research. 2005, 5:307-324, doi: https://doi.org/10.1177/ 1468794105054457

36. Midgley N. Sailing between Scylla and Charybdis: Incorporating qualitative approaches into child psychotherapy research. J Child Psychother 2004, 30:89-111, doi: http://dx.doi.org/10. 1080/0075417042000205814

37. Barus-Michel J. Le sujet social. Paris, Editions Dunod, 1987

38. Clarke S. Hoggett P. Researching beneath the surface. Karnac Books Ltd, 2009

39. Frosh S. Psychosocial studies and psychology: is a critical approach emerging? Hum Relat 2003, 56:1545-1567, doi: https:// doi.org/10.1177/00187267035612005

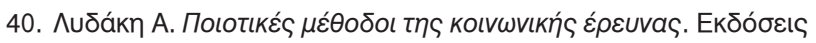
Kaotaviẃtn, 2012

41. Sherwood R. The psychodynamics of race: Vicious and benign spirals. Brighton, Harvester, 1980

42. Fine M. Disruptive voices: the possibilities for feminist research. University of Michigan Press, 2002
43. Midgley N. Psychoanalysis and qualitative psychology: Complementary or contradictory paradigms? Qualitat Research Psychol 2006, 3:213-231

44. Revault d'Allonnes C, Barus-Michel J. La psychologie sociale. Bulletin de Psychologie 1981:34

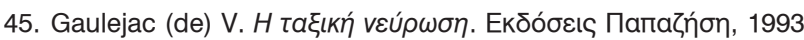

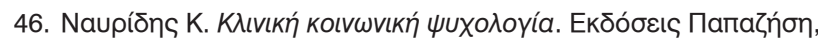
1994

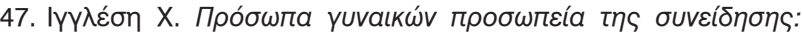

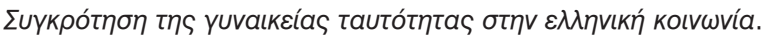

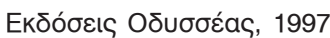

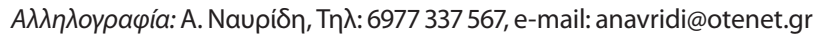

